\title{
Prognostic impact of ReTURB in high grade T1 primary bladder cancer
}

\author{
Roberto Sanseverino $^{1}$, Giorgio Napodano ${ }^{1}$, Antonio Campitelli ${ }^{1}$, Maria Addesso ${ }^{2}$ \\ ${ }^{1}$ U.O.C. di Urologia, Ospedale Umberto I, ASL Salerno, Nocera Inferiore, Italy; \\ ${ }^{2}$ U.O.C. di Anatomia Patologica, Ospedale Umberto I, ASL Salerno, Nocera Inferiore, Italy.
}

\begin{abstract}
Summary Purpose: To evaluate whether pathological outcomes of ReTURB have a prognostic impact on recurrence and progression of primitive T1HG bladder cancer.

Material and methods: Patients affected by primitive T1HG TCC of bladder underwent restaging TURB (ReTURB). Patients with muscle invasive disease at ReTURB underwent radical cystectomy; those with non-muscle invasive residual (NMI-RT) and those with no residual tumour (NRT) received an intravesical BCG therapy. We compared recurrence and progression in NMIRT patients and NRT patients at restaging TURB. Patients were followed every 3-6 months with cystoscopy and urine cytology.

Results: 212 patients were enrolled in the study. At ReTURB, residual cancer was detected in 92 of 196 (46.9\%) valuable patients: $14.3 \%$ of these were upstaged to T2. At follow up of $26.3 \pm 22.8$ months, there were differences in recurrence and progression rates between NRT and NMIRT patients: $26.9 \%$ and $45.3 \%(p<0.001), 10.6 \%$ and $23.4 \%(p 0.03)$, respectively. Recurrence-free and progression-free survivals were significantly higher in NRT compared to NMIRT patients: $73.1 \%$ and $54.7 \%(p<0.001), 89.4 \%$ and 76.6 ( $p 0.03)$, respectively. Conclusions: ReTURB allows to identify a considerable number of residual and understaged cancer. Patients with NMIRT on ReTURB have worse prognosis than those with NRT in terms of recurrence and progression free survival.

These outcomes seem to suggest a prognostic impact of findings on ReTURB that could be a valid tool in management of high grade T1 TCC.
\end{abstract}

KEY WORDS: Bladder cancer; ReTurb; Prognosis.

Submitted 20 November 2015; Accepted 11 February 2016

\author{
INTRODUCTION \\ Bladder cancer includes tumours of extremely heteroge- \\ neous biological behaviour. Approximately $75-85 \%$ of \\ all patients present with non muscle invasive (NMI) \\ bladder cancer (1). Transurethral resection of the blad- \\ der (TURB) is the cornerstone approach in the diagno- \\ sis, initial staging and therapy of transitional cell carci- \\ noma (TCC). However the rate of residual tumour after \\ TURB of neoplasms invading the lamina propria (T1 \\ TCC) ranges from $28 \%$ to $76 \%$ at any site (2-11) and \\ from 22 to $74 \%$ at the same site of first TURB (12-15). \\ Moreover 9 to $49 \%$ of tumours after TURB are under- \\ staged; particularly, up to $28 \%$ of T1 TCC are upstaged
}

to T2 at the second resection (16). High grade T1 TCC present a high risk of progression and represent a controversial therapeutic issue. It has been demonstrated that restaging TURB (ReTURB) is a valid tool to improve recurrence-free and progression-free survival, and, improving staging accuracy, to identify understaged T2 cancer that could benefit from an early radical treatment (17). However, it is not clear whether or not patients with no residual cancer at ReTURB have a better prognosis than those with residual cancer. We have evaluated the usefulness of second TURB and whether pathological outcomes of ReTURB have a prognostic impact on recurrence and progression of primitive TlG3 bladder cancer.

\section{Materials AND Methods}

Patients affected by primitive TlG3 transitional cell carcinoma of bladder were enrolled in this study and underwent second look TURB 4-6 weeks following the initial TURB Informed consent was obtained from each patient. Patients who refused ReTURB and those who have undergone incomplete resection were excluded from the study. Restaging TURB consisted of fractioned resection of all visible lesions, depth resection of base and borders of previous resection area, biopsy of any abnormal mucosal area; it was performed by the same urologist who performed the first TURB. Pathological evaluation was carried out according to the TNM system of UICC and to the WHO grading classification. Patients with muscle invasive disease underwent radical cystectomy; those with non-muscle invasive residual (NMI-RT) and those with no residual tumour (NRT) received an induction 6 weeks course of intravesical BCG followed by maintenance SWOG schedule (Table 1). All patients were followed with cystoscopy and voiding urine cytology every 3-4 months for the first and second year, every 6 months for the third and fourth year, and annually thereafter. Diagnostic imaging of the upper tract and chest $\mathrm{X}$ ray were performed at least annually or when clinically indicated. In order to evaluate the prognostic significance of ReTURB outcomes, we compared recurrence and progression rate, and recurrence and progression free survival in NMI-RT and NRT patients at restaging TURB. Recurrence was defined as first evidence of any tumour at follow up; progression was defined as 
Table 1.

Groups of patients.

\begin{tabular}{|c|l|l|}
\hline \multirow{2}{*}{ Total (196 pts) } & $\begin{array}{l}\text { No residual tumour [NRT] } \\
\text { (104 pts; 53.1\%) }\end{array}$ & \\
\cline { 2 - 3 } & $\begin{array}{l}\text { Residual tumour } \\
\text { (92 pts; 46.9\%) }\end{array}$ & $\begin{array}{l}\text { Muscle invasive residual } \\
\text { tumour (28 pts; 14.3\%) } \\
\text { Non muscle invasive } \\
\text { residual tumour [NMIRT] } \\
\text { (64 pts; 32.7\%) }\end{array}$ \\
\hline
\end{tabular}

muscle invasive tumour or evidence of metastasis. Categorical variables are presented as numbers and frequency values and compared by chi-square and Fisher exact test as appropriate. Continuous variables are expressed as mean \pm standard deviation and compared by T Student test. A cumulative survival curve for recurrence-free and progression-free survival was drawn using the Kaplan-Meier method, log-rank test was used to compare differences between NMIRT and NRT patients. A p value $\leq 0.05$ with the 2 -tailed test was considered to be statistically significant. Statistical analysis was carried out by Statistical Package for Social Sciences software package version 16.0 (SPSS, Chicago, Ill. USA).

\section{RESULTS}

From January 2002 to September 2013, 212 eligible patients were enrolled in the study. Were excluded from the study, 11 patients who refused restaging TURB and 5 who underwent incomplete resection. The average age of 196 valuable patients (173 males and 23 females) was $69.5 \pm 9.5$ years. Of the entire cohort of 196 patients, $146(74.5 \%)$ had a solitary tumour and 50 (25.5\%) had multiple lesions, at first TURB; 6 patients showed concomitant Carcinoma in Situ (CIS). In 19 patients (9.7\%) pathological analysis revealed absence of muscle in specimen. The TURB was performed by 6 experienced urologists and pathological evaluation was carried out by 1 dedicated uropathologist. At Restaging TURB residual cancer was histologically detected in 92 patients (46.9\%). In 5 cases (2.5\%) was observed prolonged bleeding ( $>24$ hours), but not requiring any blood transfusion. There was no significant statistical association between primitive tumour focality and evidence of residual cancer at ReTURB: 35.5\% (44/124) of patients with solitary tumour had residual cancer compared to $45.4 \%$

\section{Table 2.}

Histological outcomes of ReTURB.

\begin{tabular}{|lc|}
\hline Stage and grade & Pts $(\mathbf{n})$ \\
\hline T0 & 104 \\
TaG1 $(+\mathrm{CIS})$ & $6(1)$ \\
T1G1 & 4 \\
T1G2 $(+\mathrm{CIS})$ & $1(2)$ \\
TaG3 & 1 \\
T1G3 $(+\mathrm{CIS})$ & $39(2)$ \\
CIS & 8 \\
T2 & 28 \\
\hline
\end{tabular}

Table 3.

Outcomes of NRT and NMIRT patients.

\begin{tabular}{|l|c|c|c|}
\hline & NRT & NMIRT & p value \\
\hline Pts $(n)$ & 104 & 64 & \\
Age (years) & $69.3 \pm 9.9$ & $70.6 \pm 9.0$ & 0,38 \\
Mean follow up (months) & $34.8 \pm 24.0$ & $22.8 \pm 15.6$ & 0.005 \\
Multifocality at first TURB (\%) & 23.1 & 31.2 & 0.28 \\
Recurrence rate \% (n) & $26.9(28 / 104)$ & $45.3 \%(29 / 64)$ & $<0.001$ \\
Progression rate \% (n) & $10.6(11 / 104)$ & $23.4(15 / 64)$ & 0.03 \\
\hline
\end{tabular}

(20/44) of patients with multiple lesions ( $p$ 0.28). Among the 92 patients with residual cancer at ReTURB, $41(20.9 \%)$ presented identical stage and grade, while 28 (14.3\%) were upstaged to T2. Histological outcomes of the remaining patients with residual cancer are listed in Table 2.

Patients with muscle invasive cancer underwent radical cystectomy. Of the remaining 168 patients with no residual tumour (NRT) and those with non muscle invasive residual tumour (NMIRT), 57 (33.9\%) had recurrence and $26(15.5 \%)$ progressed to muscle invasive disease after a mean follow up of $26.3 \pm 22.8$ months. There was statistically significant difference in recurrence rate between NRT patients and NMIRT patients: $26.9 \%$ vs $45.3 \%$ respectively $(p<0.001)$ (Table 3$)$. Estimated actuarial recurrence-free survival rate was significantly higher in NRT group (73.1\%) compared to NMIRT patients (54.7\%) (log-rank 10.64; p < 0.001) (Figure 1). Progression of the disease occurred in $10.6 \%$ of NRT patients and in $23.4 \%$ of NMIRT patients ( $p=0.03$ ); estimated actuarial progression-free survival rate was higher in NRT group (89.4\%) compared to NMIRT patients (76.6\%) (log-rank 4.58; $p=0.03$ ) (Figure 2). Follow up of NRT patients was significantly longer than that of NMI-RT patients $(34.8( \pm 24.0)$ vs $22.8( \pm 15.6)$ months, $\mathrm{p}=0.005$ ).

\section{Discussion}

T1HG TCC are high risk tumours and they represent a great challenge for the urologists; they can present with various biological behaviour, so it is not easy to identify those with worse prognosis that could benefit by early radical cystectomy. Recurrence within 3 months of TURB is one of the most important prognostic factors for time to progression and progression free survival of NMI bladder cancer $(18,19)$. This rate depends on several factors such as stage, tumour size, number of lesions, use of adjuvant therapy, surgeon's experience (20). However it is essential to distinguish between patients with true early recurrence and those with residual tumour due to an incomplete resection. It has been demonstrated that residual cancer after TURB of $\mathrm{T} 1$ bladder cancer ranges from 28 to $76 \%$ at any site (2-11) and from 22 to $74 \%$ at the same site of first TURB (12-15), depending on stage, grade, multiplicity, appearance of tumour, surgeon's experience and modality of resection $(2,3,10,11,21)$. Moreover several studies, assessing the staging value of ReTURB, proved that 9-49\% of NMI bladder cancer had been underestimated at first $\operatorname{TURB}(3,4,7,8)$, and, par- 
Figure 1.

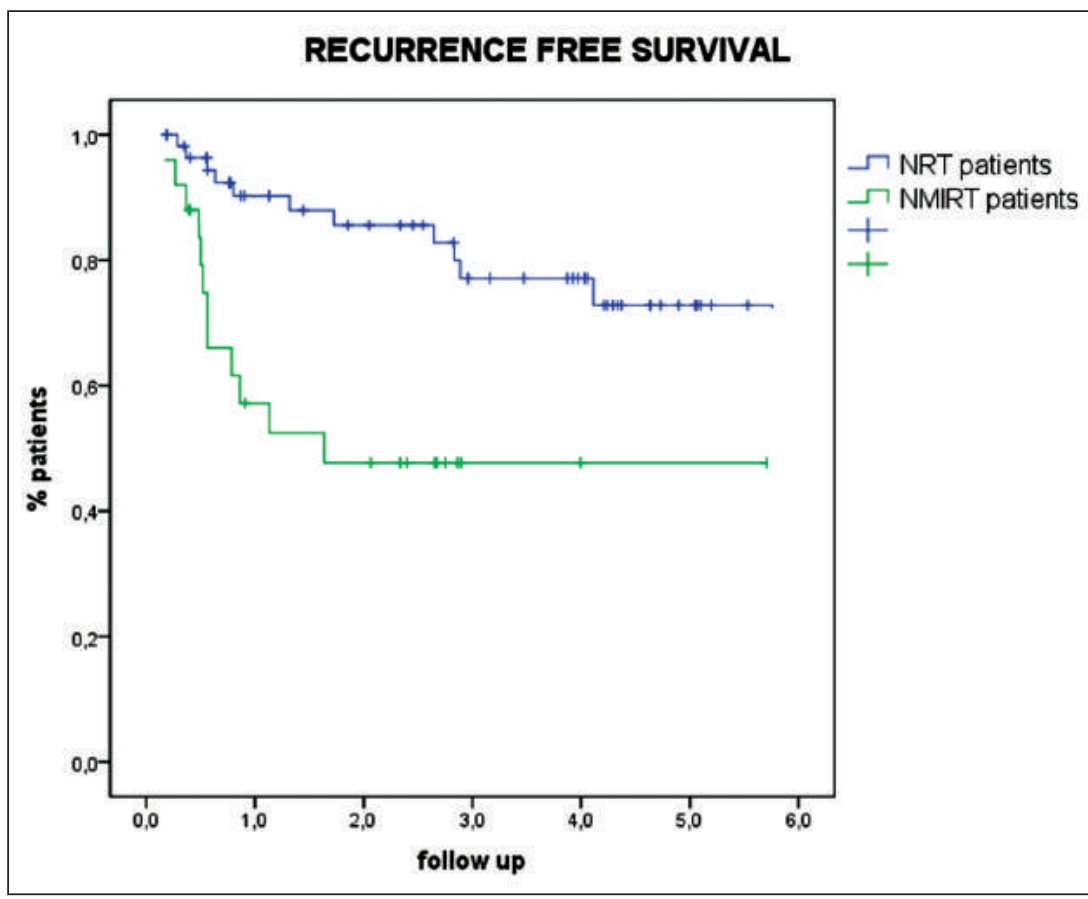

Figure 2.

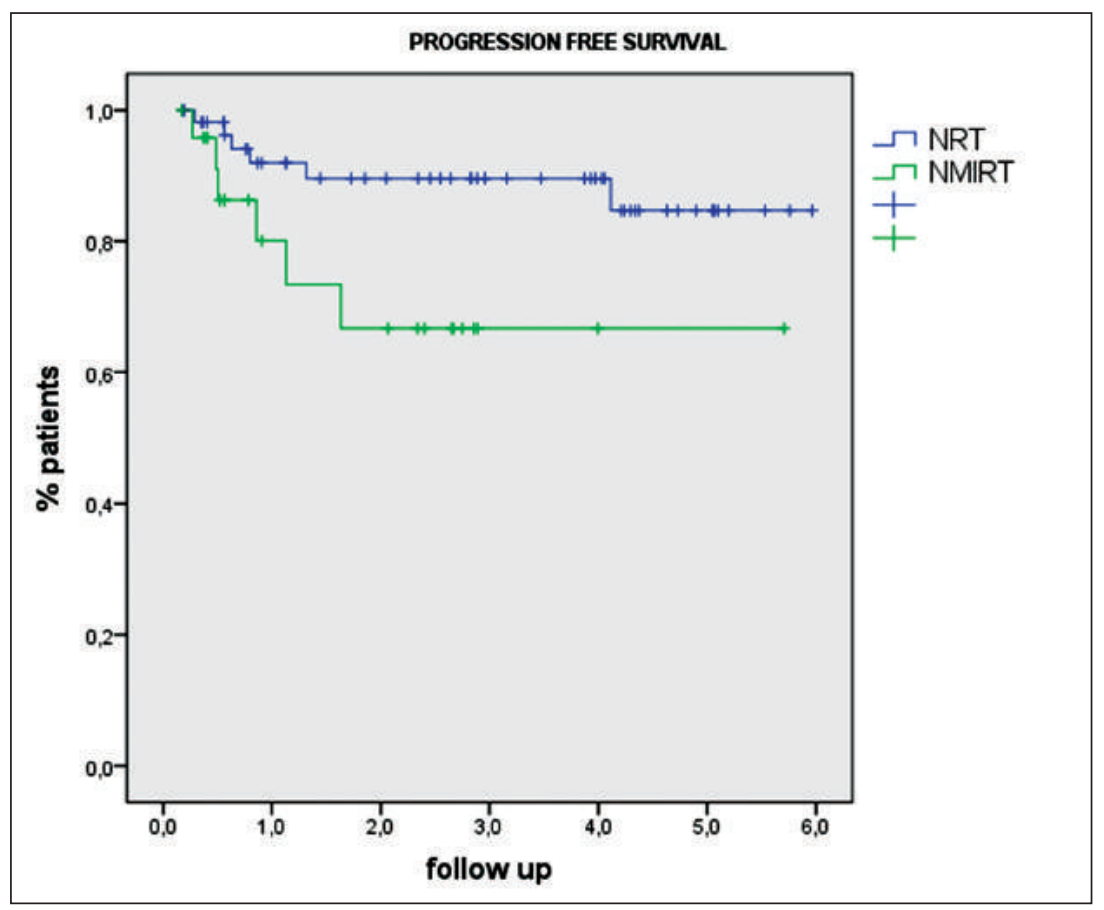

TURB for T1G3 TCC, the specimens were suitable for complete pathological assessment (26). In the study of Bernardini et al. muscularis propria was absent in 55 of 149 (36\%) patients (27); likewise Cheng et al. reported absence of muscle in TURB samples in $66 \%$ of patients with $\mathrm{T} 1$ TCC (28). Herr et al. demonstrated understaging in $49 \%$ of $\mathrm{Tl}$ patients without and in 14\% of those with muscularis propria in the specimens of first TURB. In this study $8 \%$ of $\mathrm{Ta} / \mathrm{C}$ is tumours and $27.6 \%$ of $\mathrm{Tl}$ were upstaged to muscle invasive cancer by ReTURB (29).

In our study, we have evaluated the usefulness and the prognostic significance of second TURB in patients affected by primitive high grade Tl TCC.

The second TURB was safe and only 5 self-limiting bleeding occurred.

However, though resection of bladder was performed by experienced surgeons, residual cancer occurred in $46.9 \%$ of patients. Histological evaluation revealed muscle invasive disease in $28(14.3 \%)$ patients and CIS in 13 (6.6\%). Particularly, 6 of 19 (31.6\%) patients without muscularis propria in samples of first TURB were classified as T2 TCC on second TURB; these data underline relevance of adequate samples containing muscularis propria, as yet demonstrated in other series.

The prognostic value of second look TURB on natural history of bladder cancer has not enough been elucidated; in fact, only few studies have investigated impact of pathological outcomes of ReTURB on recurrence and progression of primitive $\mathrm{Tl}$ bladder cancer. In a study on 42 patients affected by primitive T1G2-3 TCC, restaging TURB revealed $64 \%$ of residual tumours, $4.8 \%$ of muscle invasive cancers and $19 \%$ of CIS. At mean follow up of 60 months the recurrence rate of no residual tumour (NRT) patients was 33\% compared to $57 \%$, $75 \%$ and $87.5 \%$ of $\mathrm{Ta}, \mathrm{Cis}$ and $\mathrm{T} 1$ residual cancer ( $\mathrm{Tr}$ ) patients, respectively; moreover, organ preservation rate was $100 \%$ for NRT patients and

ticularly, up to $28 \%$ of T1 TCC had been upstaged to T2 by second TURB (16). The accuracy of histological evaluation depends on several factors related to cancer characteristics, experience of pathologist and quality of samples $(24,25)$. For a thorough and accurate histological evaluation it is necessary to achieve adequate specimens containing muscularis propria. Mulders et al. showed that in only 121 of 155 (78\%) patients, who underwent
$64 \%$ for Tr patients (8). In a prospective randomized trial, Divrik et al. compared outcomes of ReTURB plus intravesical MMC (Group 1) with TURB plus intravesical MMC (Group 2) in 210 patients affected by T1 TCC of bladder; at mean follow up of 66.1 months, recurrence rate was $37 / 93 \%$ in group 1 and 70/98 2\% in group 2. Median recurrence free survival (RFS) was 47 months for group 1 compared to 12 months for group 2. Progression was 
observed in $6.5 \%$ of patients for group 1 and in $23.5 \%$ of patients for group $2(\mathrm{p}=0.001)$. Median progression free survival (PFS) was 73 months for group 1 compared to 53.5 months for group 2. Overall survival of two groups was $67.7 \%$ and $64.3 \%$ (log rank 0.363 ), respectively. This study demonstrated that the differences in terms of recurrence between two groups were due to the presence of residual tumour in group 2 rather than true recurrence and that intravesical chemotherapy did not compensate for inadequate resection (17).

In a recent study on $352 \mathrm{Tl}$ TCC patients with a 7.5 years of median follow up, those with T1 residual cancer on ReTURB presented higher BCG failure rate (53\%), recurrence rate (88\%) and progression rate $(82 \%)$ than patients with no residual cancer $(3 \%, 48 \%$ and $8 \%$, respectively). The authors suggest that early cystectomy may be advised for patients with $\mathrm{Tl}$ residual cancer on Restaging TURB (30).

Our data revealed a statistical significant difference in recurrence rate, recurrence free survival, progression rate and progression free survival between patients with and without residual cancer on ReTURB. Patients with no muscle invasive residual tumour on ReTURB have higher recurrence (45.3\% vs $26.9 \%$ ), progression rates (23.4\% vs $10.6 \%)$ and worse recurrence and progression free survival than those with no residual tumour, though they presented significant shorter follow up (22.8 vs 34.8 months).

These outcomes seem to suggest a prognostic impact of findings on ReTURB that could be a valid tool in identify patients at high risk of progression that could be ideal candidates for an early radical cystectomy. However, evidences of our study are limited by retrospective data evaluation and relatively short follow up.

\section{Conclusions}

Complete tumour eradication and correct staging are of paramount importance in primary diagnosis and treatment of bladder cancer. It has been clearly shown that persistence of tumour can negatively affect recurrence and even progression free survival. Understaging with delay of appropriate treatment can affect overall patient survival after radical cystectomy.

TURB still remains the cornerstone modality for staging and primary treatment of bladder cancer. However, even in experienced hands, it is far from being perfect, with a consistent percentage of residual disease or tumour understaging left behind. Second look TURB in a short delay appears to be very useful in case of $\mathrm{Tl}$ and/or high grade tumours and mandatory whenever the tissue specimen does not allow a correct evaluation of the muscular layer. In our study patients with non muscle invasive residual tumour on ReTURB show worse prognosis than those with no residual tumour in terms of recurrence and progression of disease.

A longer follow up and a larger series of patients are essential to confirm the prognostic value of findings on ReTURB and to demonstrate whether ReTURB outcomes can identify patients at high risk of progression that could benefit from early cystectomy.

\section{REFERENCES}

1. Oosterlinck W, van der Meijden A, Sylvester R, et al. Guidelines on Ta-T1 (Non muscle-invasive) bladder cancer. In: Guidelines, European Association of Urology 2006; pp 3-17.

2. Klan R, Loy V, Huland $H$. Residual tumor discovered in routine second transurethral resection in patients with stage T1 transitional cell carcinoma of the bladder. J Urol. 1991; 146:316-8.

3. Mersdorf A, Brauers A, Wolff JM, et al. 2nd TURB for superficial bladder cancer: a must? J Urol 1998; 159:143, abstract 542.

4. Herr HW. The value of a second transurethral resection in evaluating patients with bladder tumors. J Urol. 1999; 162:74.

5. Schips L, Augustin H, Zigeuner RE, et al. Is repeated transurethral resection justified in patients with newly diagnosed superficial bladder cancer? Urology 2002; 59:220.

6. Grimm M-O, Steinhoff C, Simon X, et al. Effect of routine repeat transurethral resection for superficial bladder cancer: a long-term observational study. J Urol. 2003; 170:433.

7. Vögeli TA, Grimm M-O, Ackermann R. Prospective study for quality control of TURB of bladder tumors by routine 2 nd TURB (ReTURB). J Urol. 1998; 159:143, abstract 543.

8. Brauers A, Buettner, R, Jakse, G. Second resection and prognosis of primary high risk superficial bladder cancer: is cystectomy often too early? J Urol. 2001; 165:808.

9. Rigaud J, Karam G, Braud, G, et al. T1 bladder tumors: value of a second endoscopic resection. Prog Urol. 2002; 12:27.

10. Schwaibold $H$, Treiber $U$, Kubler $H$, et al. Significance of 2 nd transurethral resection for T1 bladder cancer. Eur Urol. 2000; 37 (Suppl 2):11.

11. Divrik T, Yildirim U, Eroglu A, et al. Is a second transurethral resection necessary for newly diagnosed $\mathrm{p} T 1$ bladder cancer? J Urol. 2006; 175:1258-61

12. Jakse G, Loidl W, Seeber G, Hofstadter F. Stage T1, grade 3 transitional cell carcinoma of the bladder: an unfavourable tumor? J Urol. 1987; 137:39-43.

13. Lundbeck F, Jacobsen T, Vaeth M. Impact of the category of early tumor recurrence on tumor progression in bladder tumors treated by transurethral resection. Eur Urol. 1989; 16:291-4.

14. Wolf H, Iversen HG, Rosenkilde P, Schroder T. Transurethral surgery in the treatment of invasive bladder cancer (T1 and T2). Scand J Urol Nephrol. 1987; 104:127-32.

15. Schwaibold H, Sivalingam S, May F, Hartung R. The value of a second transurethral resection for $T 1$ bladder cancer. Brit J Urol Int. 2006; 97:1199-201.

16. Miladi M, Peyromaure M, Zerbib M, et al. The value of a second transurethral resection in evaluating patients with bladder cancer. Eur Urol. 2003; 43:241-5.

17. Divrik T, Sahin A, Yildirim U, et al. Impact of routine second Transurethral Resection on the long-term outcome of patients with newly diagnosed PT1 Urothelial Carcinoma with respect to recurrence, progression rate, and disease-specific survival: a prospective randomised clinical trial. Eur Urol. 2010; 58:185-90.

18. Fitzpatrick JM, West AB, Butler MR, et al. Superficial bladder tumors: the importance of recurrence pattern following initial resection. J Urol. 1986; 135:920-4.

19. Kurth KH, Schroeder FH, Debruyne F, et al. Long-term followup in superficial transitional cell carcinoma of the bladder: prognostic factors for time to first recurrence, recurrence rate, and survival. 
Final results of a randomized trial comparing doxorubicin hydrochloride, ethoglucid, and transurethral resection alone. EORTC Genitourinary Tract Cancer Cooperative Group. Prog Clin Biol Res 1989; 303:481.

20. Brausi M, Collette L, Kurth K, et al. Variability in the recurrence rate at first follow up cystoscopy after TURB in stage Ta T1 transitional cell carcinoma of the bladder: a combined analysis of seven EORTC studies. Eur Urol 2002; 41: 523-31.

21. Kohrmann KU, Woeste M, Kappers JH, Rassweiler J, Alken P. Der Wert der transurethralen Nachresektion beim oberflachlichen Harnblasenkarzinom. Akt Urol. 1994; 25:208-13.

22. Zurkirchen MA, Sulser T, Gaspert A, Hauri D. Second transurethral resection of superficial transitional cell carcinoma of the bladder: a must even for experienced urologists. Urol Int. 2004; 72:99102.

23. Pathologists of the French Association of Urology Cancer Committee. Lamina propria microinvasion of bladder tumors, incidence or stage allocation ( $\mathrm{TTa}$ vs $\mathrm{pT1}$ ): recommended approach. World J Urol. 1993; 11:161-4.

24. Grigor KM, Bollina. Interpathologist variartion in the assess- ment of G3pT1 bladder carcinoma. Eur Urol. 1996; 30 (Suppl 2):A850.

25. Witjes JA, Kiemeney LA, Schaafsma HE, Debruyne FM. The influence of review pathology on study outcome of a randomized multicenter suprerficial bladder cancer trial. Br J Urol. 1994; 73:172-6.

26. Mulders PFA, Hoekstra WJ, Heybroek RPM, et al. Prognosis and treatment of T1G3 bladder tumours. A prognostic factor analysis of 121 patients. Eur J Cancer 1994; 30:914-7.

27. Bernardini S, Billerey $C$, Martin $M$, et al. The predictive value of muscularis mucosae invasion and p53 over expression on progression of stage $T 1$ bladder carcinoma. J Urol. 2001; 165:42-6.

28. Cheng L, Neumann RM, Weaver AL, et al. Grading and staging of bladder carcinoma in transurethral resection specimens. Am J Clin Pathol. 2000; 113:275-9.

29. Herr HW. Does cystoscopy correlate with histology of recurrent papillary tumours of the bladder? BJU Int. 2001; 88:83-685.

30. Herr HW, Donat SM, Dalbagni G. Can restaging transurethral resection of $\mathrm{T1}$ bladder cancer select patients for immediate cystectomy? J Urol 2007; 177:75-9.

\section{Correspondence}

Roberto Sanseverino, MD

roberto.sanseverino@libero.it

Giorgio Napodano, MD

giorgio.napodano@gmail.com

Antonio Campitelli, MD

U.O.C. di Urologia, Ospedale Umberto I, ASL Salerno

Nocera Inferiore, Italy

Maria Addesso, MD

U.O.C. di Anatomia Patologica, Ospedale Umberto I, ASL Salerno

Nocera Inferiore, Italy 